\title{
Association of depressive disorders, depression characteristics and antidepressant medication with inflammation
}

\author{
N Vogelzangs ${ }^{1}$, HE Duivis ${ }^{2}$, ATF Beekman ${ }^{1}$, C Kluft ${ }^{3}$, J Neuteboom ${ }^{3}$, W Hoogendijk ${ }^{4}$, JH Smit ${ }^{1}$, P de Jonge ${ }^{5}$ and BWJH Penninx ${ }^{1,5,6,7}$
}

Growing evidence suggests that immune dysregulation may be involved in depressive disorders, but the exact nature of this association is still unknown and may be restricted to specific subgroups. This study examines the association between depressive disorders, depression characteristics and antidepressant medication with inflammation in a large cohort of controls and depressed persons, taking possible sex differences and important confounding factors into account. Persons (18-65 years) with a current $(N=1132)$ or remitted $(N=789)$ depressive disorder according to DSM-IV criteria and healthy controls $(N=494)$ were selected from the Netherlands Study of Depression and Anxiety. Assessments included clinical characteristics (severity, duration and age of onset), use of antidepressant medication and inflammatory markers (C-reactive protein (CRP), interleukin-6 (IL-6), tumor necrosis factor-alpha (TNF- $\alpha$ )). After adjustment for sociodemographics, currently depressed men, but not women, had higher levels of CRP (1.33 versus $0.92 \mathrm{mg} \mathrm{I}^{-1}, P<0.001$, Cohen's $\mathrm{d}=0.32$ ) and IL-6 ( 0.88 versus $0.72 \mathrm{pg} \mathrm{ml}^{-1}, P=0.01$, Cohen's $d=0.23$ ) than non-depressed peers. Associations reduced after considering lifestyle and disease indicators especially body mass index - but remained significant for CRP. After full adjustment, highest inflammation levels were found in depressed men with an older age of depression onset (CRP, TNF- $\alpha$ ). Furthermore, inflammation was increased in men using serotonin-norepinephrine reuptake inhibitors (CRP, IL-6) and in men and women using tri- or tetracyclic antidepressants (CRP), but decreased among men using selective serotonin reuptake inhibitors (IL-6). In conclusion, elevated inflammation was confirmed in depressed men, especially those with a late-onset depression. Specific antidepressants may differ in their effects on inflammation.

Translational Psychiatry (2012) 2, e79; doi:10.1038/tp.2012.8; published online 21 February 2012

\section{Introduction}

Depression is a complex heterogeneous disorder, which may need a similarly heterogeneous offer of treatment possibilities. Currently available antidepressant medications largely target monoamine pathways, but treatment of depression is only effective in about a third to a half of patients., Identification of additional pathophysiological pathways involved in depression (subtypes) is needed to guide the development of alternative treatment strategies. Increasing interest has been directed to immune dysregulation in depression. Recently, two meta-analyses have shown that inflammatory marker levels such as $C$-reactive protein (CRP), interleukin (IL)-6 and tumor necrosis factor alpha (TNF- $\alpha$ ) are increased in depressed persons compared with nondepressed subjects. ${ }^{3,4}$

Although the results of these meta-analyses are promising, the evidence for immune dysregulation in depression is not conclusive. A substantial portion of studies included in these meta-analyses did not adequately adjust for possible confounding factors. The association between depression and inflammation appeared much smaller, although still present, in studies adjusting for body mass index (BMI). ${ }^{4}$ The effect estimate after a more complete adjustment (including several lifestyle and disease factors) is not entirely clear. Also, most of the larger studies examining depression and inflammation have used depressive symptoms questionnaires instead of assessing psychiatric diagnoses by means of clinical interviews. The former is much more prone to confounding by somatic health as a person can score high on these questionnaires based on having many physical complaints. Furthermore, a large part of previous studies has been conducted within older populations. More studies within younger age samples are therefore needed.

Next to these general limitations, meta-analyses have found very large heterogeneity across studies. ${ }^{3,4}$ It is plausible that immune dysregulation is not generally present in depression, but restricted to particular subgroups of

\footnotetext{
${ }^{1}$ Department of Psychiatry and EMGO Institute for Health and Care Research, VU University Medical Center, Amsterdam, The Netherlands; ${ }^{2}$ Center of Research on Psychology in Somatic Diseases, Tilburg University, Tilburg, The Netherlands; ${ }^{3}$ Good Biomarker Sciences, Leiden, The Netherlands; ${ }^{4}$ Department of Psychiatry, Erasmus Medical Center, Rotterdam, The Netherlands; ${ }^{5}$ Department of Psychiatry, University Medical Center Groningen, Groningen, The Netherlands; ${ }^{6}$ Department of Psychiatry, Leiden University Medical Center, Leiden, The Netherlands and ${ }^{7}$ Neuroscience Campus Amsterdam, VU University Medical Center, Amsterdam, The Netherlands

Correspondence: Dr N Vogelzangs, Department of Psychiatry and EMGO Institute for Health and Care Research, VU University Medical Center, AJ Ernststraat 1187, Amsterdam $1081 \mathrm{HL}$, The Netherlands.
}

E-mails: n.vogelzangs@vumc.nl and n.vogelzangs@ggzingeest.nl

Keywords: antidepressants; cohort study; depression characteristics; depressive disorder; inflammation

Received 13 October 2011; revised 23 December 2011; accepted 8 January 2012 
depressed persons. Several factors that could influence the depression-inflammation relationship need further investigation to help delineate the depressed person with immune dysregulation. This is important to give direction to whom new treatment strategies could be targeted.

First, sex differences might exist, but results are thus far inconsistent. Stronger, weaker or similar effects have been found for women compared with men for different inflammatory markers. ${ }^{4,5}$ Inflammation levels fluctuate throughout female life according to hormonal changes owing to phase of menstrual cycle, use of hormonal contraceptives, menopause and use of estrogens, ${ }^{6-8}$ which might influence the relationship between depression and inflammation.

Second, it is largely unknown whether specific depression characteristics indicate immune dysregulation. ${ }^{3}$ In line with a dose-response assumption, more severe and/or more chronic disorders can be hypothesized to show the most inflammation. ${ }^{9,10}$ In addition, late-onset depression has been associated with family history of vascular disease ${ }^{7}$ and atherosclerosis. ${ }^{11,12}$ As immune dysregulation is critically involved in vascular disease, ${ }^{13}$ it can be hypothesized that increased inflammation is specifically present in those with late-onset depressive disorders.

Third, antidepressant medication might influence inflammation levels and this effect might differ across type of medication. As summarized by Miller et al., ${ }^{14}$ several studies showed that antidepressant treatment, mainly selective serotonin reuptake inhibitors, was associated with decreases in inflammatory markers. In contrast, recent results of two large studies suggest that use of antidepressants, mainly tricyclic antidepressants (TCA), is associated with elevated inflammation levels. ${ }^{15}$

To address the issues raised above, the first aim of the present study was to examine the association between diagnosed depressive disorders and inflammatory markers (CRP, IL-6 and TNF- $\alpha$ ), using a large and relatively young cohort of depressed persons and controls, taking possible sex differences and important confounding factors into account. The second aim was to investigate whether specific depression characteristics (severity, duration and age of onset) could further delineate the depressed person with increased inflammation. Lastly, possible effects of (specific types of) antidepressant medication on inflammation levels were examined.

\section{Subjects and methods}

Sample. The Netherlands Study of Depression and Anxiety (NESDA) is an ongoing cohort study designed to investigate the long-term course and consequences of depressive and anxiety disorders. Participants were 18 to 65 years old at baseline assessment in 2004-2007 and were recruited from the community (19\%), general practice (54\%) and secondary mental health care (27\%). A total of 2981 persons were included, consisting of persons with a current or past depressive and/or anxiety disorder and healthy controls. A detailed description of the NESDA study design and sampling procedures can be found elsewhere. ${ }^{16}$ The research protocol was approved by the Ethical Committee of participating universities, and after complete description of the study all respondents provided written informed consent.

During the baseline interview, presence of depressive disorder (major depressive disorder, dysthymia) and anxiety disorder (social phobia, generalized anxiety disorder, panic disorder and agoraphobia) was established using the Composite Interview Diagnostic Instrument (CIDI) according to DSM-IV criteria. $^{17}$ The CIDI is a highly reliable and valid instrument for assessing depressive and anxiety disorders ${ }^{18}$ and was administered by specially trained research staff. In addition, the severity of depression was measured in all participants using the 28-item self-report Inventory of Depressive Symptoms (IDS). ${ }^{19}$ For the present analyses we selected persons with a current (that is, past 6 months; $N=1158$ ) or remitted (lifetime, but not current; $N=815$ ) depressive disorder and healthy controls without any lifetime depressive or anxiety disorder and an IDS score below 14 $(N=506)$. Of these 2479 persons, 64 were excluded because of missing information on inflammatory markers, leaving a sample of 2415 persons for the present study. Persons with missing data on inflammation did not differ from included persons in terms of sex, age, years of education and depressive disorder status.

Inflammatory markers. Markers of inflammation were assessed at the baseline NESDA measurement and included CRP, IL- 6 and TNF- $\alpha$. Fasting blood samples of NESDA participants were obtained in the morning around 0800 hours and kept frozen at $-70^{\circ} \mathrm{C}$. CRP and IL- 6 were assayed at the Clinical Chemistry department of the VU University Medical Center. High-sensitivity plasma levels of CRP were measured in duplicate by an in-house ELISA based on purified protein and polyclonal anti-CRP antibodies (Dako, Glostrup, Denmark). Intra- and inter-assay coefficients of variation were $5 \%$ and $10 \%$, respectively. Plasma IL-6 levels were measured in duplicate by a high sensitivity ELISA (PeliKine Compact ELISA, Sanquin, Amsterdam, The Netherlands). Intra- and inter-assay coefficients of variation were $8 \%$ and $12 \%$, respectively. Plasma TNF- $\alpha$ levels were assayed in duplicate at Good Biomarker Science (Leiden, The Netherlands), using a highsensitivity solid phase ELISA (Quantikine HS Human TNF- $\alpha$ Immunoassay, R\&D systems, Minneapolis, MN, USA). Intraand inter-assay coefficients of variation were $10 \%$ and $15 \%$, respectively.

Depression characteristics. Next to CIDI depressive disorder diagnosis, depression characteristics included depressive symptoms severity as measured by the IDS, and depressive symptoms duration, using the Life Chart method, ${ }^{20}$ in which a detailed account of the presence of depressive symptoms during the past 4 years was assessed. From this, the percent of time patients reported depressive symptoms was computed. Additionally, age of depression onset was derived from the CIDI interview.

Antidepressant medication. Medication use was assessed based on drug container inspection of all drugs used in the past month and classified according to the World Health Organization Anatomical Therapeutic Chemical 
classification. ${ }^{21}$ Antidepressant medication was only considered when taken on a regular basis (at least $50 \%$ of the time) and included selective serotonin reuptake inhibitors (SSRI; N06AB), serotonin-norepinephrine reuptake inhibitors (SNRI; N06AX16 and N06AX21), TCA (N06AA) and tetracyclic antidepressants (TeCA; N06AX03, N06AX05 and N06AX11).

Covariates. Sociodemographic characteristics included age, sex and years of education. As lifestyle characteristics can be associated with both depression and inflammation, smoking status (never, former and current; assessed by selfreport), alcohol intake (<1, 1-14 (women) / 1-21 (men), >14 (women)/>21 (men) drinks per week; based on general guidelines that are used in health organizations in the Netherlands ${ }^{22}$ and as used in other studies ${ }^{23}$ ), BMI (weight in kilograms divided by height in meters squared) and physical activity (measured with the International Physical Activity Questionnaire ${ }^{24}$ in MET-minutes (ratio of energy expenditure during activity compared with rest times the number of minutes performing the activity) per week) were assessed. In addition, several disease related covariates were taken into account including presence of cardiovascular disease (assessed by self-report supported by appropriate medication use (see ref. 25 for detailed description), presence of diabetes (fasting plasma glucose level $\geqslant 7.0 \mathrm{mmol} \mathrm{I}^{-1}$ or use of anti-diabetic medication (A10)), and the number of other self-reported chronic diseases for which persons received treatment (including lung disease, osteoarthritis or rheumatic disease, cancer, ulcer, intestinal problem, liver disease, epilepsy and thyroid gland disease). As suggested by Howren et al., ${ }^{4}$ to control for possible medication effects, we assessed statin use (C10AA, C10B) and use of systemic anti-inflammatory medication (M01A, M01B, A07EB and A07EC). In women, we additionally assessed use of sex hormones (self-reported use of oral contraceptives or ATC code G03), self-reported menstrual cycle phase (menstrual (0-3 days since start last menstruation), follicular (4-13 days), luteal (14-32 days)) and self-reported postmenopausal status (yes/no).

Statistical analyses. All analyses were conducted using SPSS version 15.0 statistical software (SPSS Inc., Chicago, IL, USA). Differences in baseline characteristics across sex were tested for statistical significance using $\chi^{2}$-statistics for dichotomous and categorical variables and independent $t$ tests and Mann-Whitney $U$-tests for normally and nonnormally distributed continuous variables. For subsequent analyses, CRP, IL- 6 and TNF- $\alpha$ were In-transformed to normalize distributions and presented back-transformed. Associations between baseline characteristics and inflammatory markers were tested using independent samples $t$ tests for dichotomous variables, one-way analyses of variance for categorical variables and Pearson's correlations for continuous variables.

Associations between depressive disorders and inflammatory markers were examined using analyses of (co)variance and (adjusted) means across depression groups (no, remitted and current) were presented. To take the effects of important confounding factors into account, three different models were tested: unadjusted, adjusted for sociodemographics (sex, age and education) and additionally adjusted for lifestyle and disease (smoking status, alcohol intake, BMI, physical activity, cardiovascular disease, diabetes, number of other chronic diseases, statins and anti-inflammatory medication). To investigate possible sex differences, we tested for sex interactions by including a sex-depressive disorder interaction term. When present, analyses were repeated sex stratified. For significant associations, Cohen's d was calculated in order to assess effect size.

To test whether specific depression characteristics were related to elevated inflammation levels, we performed linear regression analyses for each depression characteristic within the sample of persons with a current depressive disorder. Sex interactions were again tested and if present, shown sex stratified.

To investigate possible effects of antidepressant medications, adjusted means of inflammation levels across different medication groups were calculated using analyses of covariance and presented sex stratified in case sex interaction was present.

\section{Results}

Baseline characteristics of the total sample and for men $(N=800)$ and women $(N=1615)$ separately are shown in Table 1. Women were younger, less often smokers, more often non-drinkers, had a lower BMI, less often cardiovascular disease or diabetes and less often used statins than men. In addition, women had higher levels of CRP, but lower levels of IL- 6 and TNF- $\alpha$ than men. In men, older age, less education, smoking, heavy or non-drinking, higher BMI, lower physical activity, cardiovascular disease, diabetes, higher number of other chronic diseases, statin use and use of anti-inflammatory medication were associated with higher levels of at least one of the inflammatory markers (data not shown). In women, similar associations were found, except for smoking, heavy drinking, and physical activity. The Pearson's correlations between inflammatory markers were modest, likely reflecting only partial biological overlap, and somewhat higher in men (CRP-IL-6: $\quad r=0.40 ; \quad$ CRP-TNF- $\alpha$ : $r=0.22$; IL-6-TNF- $\alpha$ : $r=0.17$ ) than in women (CRP-IL-6: $r=0.28$; CRP-TNF- $\alpha$ : $r=0.10$; IL-6-TNF- $\alpha: r=0.09$ ).

Table 2 shows (adjusted) mean inflammation levels across depression groups (controls, remitted and current depressive disorder) based on analyses of (co) variance. In the total sample, current depressive disorders were significantly associated with higher levels of CRP and IL-6 in the unadjusted model. After additional adjustment for sociodemographics, lifestyle and disease factors, these associations disappeared. No associations were found for TNF- $\alpha$. For current depressive disorders, sex interactions were found for CRP $(P$-interaction $<0.001)$ and IL-6 $(P$-interaction $=0.009)$, but not TNF- $\alpha(P$-interaction $=0.99)$.

In men, after adjustment for sociodemographics, a current depressive disorder was associated with higher CRP levels (1.33 versus $0.92 \mathrm{mgl}^{-1}, P<0.001$, Cohen's $\mathrm{d}=0.32$ ) and IL-6 levels (0.88 versus $0.72 \mathrm{pg} \mathrm{ml}^{-1}, P=0.01$, Cohen's $\mathrm{d}=0.23$ ). Even after full adjustment for lifestyle and disease factors, men with a current depressive disorder had higher 


\begin{tabular}{|c|c|c|c|c|}
\hline & $\begin{array}{l}\text { Total sample } \\
(\mathbb{N}=2415)\end{array}$ & $\begin{array}{c}\text { Men } \\
(\mathrm{N}=800)\end{array}$ & $\begin{array}{c}\text { Women } \\
(N=1615)\end{array}$ & $\mathbf{P}^{\mathbf{a}}$ \\
\hline \multicolumn{5}{|l|}{ Sociodemographics } \\
\hline $\begin{array}{l}\text { Age (years), mean (s.d.) } \\
\text { Education (years), mean (s.d.) }\end{array}$ & $\begin{array}{l}41.8(12.9) \\
12.2(3.3)\end{array}$ & $\begin{array}{l}43.7(12.6) \\
12.0(3.3)\end{array}$ & $\begin{array}{l}40.9(12.9) \\
12.2(3.3)\end{array}$ & $\begin{array}{c}<0.001 \\
0.12\end{array}$ \\
\hline \multicolumn{5}{|l|}{ Lifestyle and Disease } \\
\hline Smoking status & & & & 0.003 \\
\hline Never, \% & 27.9 & 23.5 & 30.1 & \\
\hline Former, \% & 32.7 & 34.3 & 31.9 & \\
\hline Current, \% & 39.4 & 42.3 & 38.0 & \\
\hline Alcohol intake & & & & $<0.001$ \\
\hline$<1$ Drink a week, $\%$ & 32.9 & 22.9 & 37.8 & \\
\hline $1-14$ (women) / 1-21 (men) drinks a week, \% & 55.9 & 66.5 & 50.7 & \\
\hline$>14$ (women) / > 21 (men) drinks a week, \% & 11.2 & 10.6 & 11.5 & \\
\hline Body mass index, mean (s.d.) & $25.7(5.1)$ & $26.3(4.6)$ & $25.4(5.3)$ & $<0.001$ \\
\hline Physical activity (MET-min per week), mean (s.d.) & $3673(3026)$ & $3758(3324)$ & $3630(2867)$ & 0.36 \\
\hline Cardiovascular disease, $\%$ & 5.6 & 9.4 & 3.8 & $<0.001$ \\
\hline Diabetes, \% & 4.9 & 7.8 & 3.5 & $<0.001$ \\
\hline Number of other chronic diseases, mean (s.d.) & $0.4(0.7)$ & $0.4(0.7)$ & $0.4(0.7)$ & 0.11 \\
\hline Statin use, \% & 6.6 & 10.9 & 4.5 & $<0.001$ \\
\hline Anti-inflammatory medication use, $\%$ & 4.2 & 4.0 & 4.3 & 0.75 \\
\hline \multicolumn{5}{|l|}{ Depression characteristics } \\
\hline Depressive disorder & & & & 0.004 \\
\hline Controls, \% & 20.5 & 24.1 & 18.6 & \\
\hline Remitted depressive disorder, \% & 32.7 & 29.8 & 34.1 & \\
\hline Current depressive disorder, $\%$ & 46.9 & 46.1 & 47.2 & \\
\hline \multicolumn{5}{|l|}{ Within currently depressed cases $(N=1132)$} \\
\hline Severity (IDS score), mean (s.d.) & $32.5(12.2)$ & $32.9(12.6)$ & $32.3(12.1)$ & 0.46 \\
\hline Duration (\% of time depressed), mean (s.d.) & $38.5(30.3)$ & $40.9(31.6)$ & $37.4(29.6)$ & 0.07 \\
\hline Age of depression onset (years), mean (s.d.) & $27.1(12.5)$ & $29.7(13.3)$ & $25.9(11.8)$ & $<0.001$ \\
\hline Antidepressant use & & & & 0.13 \\
\hline No antidepressant, \% & 57.2 & 57.5 & 57.1 & \\
\hline SSRI, \% & 28.8 & 25.7 & 30.3 & \\
\hline SNRI, \% & 7.2 & 9.5 & 6.2 & \\
\hline $\mathrm{TCA}, \%$ & 3.8 & 3.5 & 3.9 & \\
\hline TeCA, \% & 2.9 & 3.8 & 2.5 & \\
\hline \multicolumn{5}{|l|}{ Inflammatory markers } \\
\hline C-reactive protein $\left(\mathrm{mg} \mathrm{I}^{-1}\right)$, median (IQR) & $1.22(0.54-3.02)$ & $1.09(0.51-2.67)$ & $1.30(0.56-3.21)$ & 0.001 \\
\hline Interleukin-6 (pg mi $\left.{ }^{-1}\right)$, median (IQR) & $0.76(0.50-1.25)$ & $0.81(0.53-1.36)$ & $0.72(0.49-1.19)$ & 0.003 \\
\hline Tumor necrosis factor-alpha $\left(\mathrm{pg} \mathrm{ml}^{-4}\right)$, median (IQR) & $0.80(0.60-1.10)$ & $0.80(0.60-1.10)$ & $0.70(0.60-1.10)$ & 0.001 \\
\hline
\end{tabular}

Abbreviations: IDS, inventory of depressive symptoms; IQR, interquartile range; s.d., standard deviation; SSRI, selective serotonin reuptake inhibitor; SNRI, serotonin-norepinephrine reuptake inhibitor; TCA, tricyclic antidepressant; TeCA, tetracyclic antidepressant.

a Based on $\chi^{2}$-test for dichotomous and categorical variables, and independent samples $t$-test for continuous variables testing the difference between men and women; for inflammatory markers the Mann-Whitney U-test was used.

CRP levels compared with controls $\left(1.29\right.$ versus $1.04 \mathrm{mgl}^{-1}$, $P=0.02$, Cohen's $\mathrm{d}=0.21$ ), and marginally higher IL-6 levels (0.87 versus $0.76 \mathrm{pg} \mathrm{ml}^{-1}, P=0.10$, Cohen's $\mathrm{d}=0.15$ ). No associations with TNF- $\alpha$ were found in men. Overall, BMI weakened the associations most, followed by number of other chronic diseases. Smoking status, alcohol use and physical activity weakened associations slightly further. Adjustment for cardiovascular disease, diabetes, statins and anti-inflammatory medication hardly affected associations.

In women, depressive disorders were not associated with inflammatory markers before or after adjustment. Although sex hormone use, menstrual cycle phase and postmenopausal status were strongly associated with inflammation levels, additional adjustment for these factors did not change the results for women. Also, there were no significant postmenopausal status-depression interactions in the associations with inflammation levels (all $P>0.40$ ) suggesting that associations between depression and inflammation appeared absent in both premenopausal $(N=1076)$ and postmenopausal women $(N=539)$.

To investigate whether specific depression characteristics (severity, duration and age of onset) were associated with higher inflammation levels, linear regression analyses were performed within the subgroup of currently depressed persons $(N=1132$; Table 3$)$. Women with more severe depressive symptoms had higher levels of TNF- $\alpha$. No associations were found for duration of depressive symptoms. Men with an older age of depression onset had higher levels of $\mathrm{CRP}$ and TNF- $\alpha$ than those with a younger age of depression onset.

Lastly, the association between antidepressant medication use and inflammation levels was examined. To incorporate possible differences in depression severity between persons who were or were not using antidepressants, we selected a 
Table 2 Adjusted mean inflammation levels across depression groups

\begin{tabular}{|c|c|c|c|c|c|c|c|c|c|}
\hline & \multicolumn{3}{|c|}{ Controls } & \multicolumn{3}{|c|}{ Remitted depressive disorder } & \multicolumn{3}{|c|}{ Current depressive disorder } \\
\hline & Mean & (s.e.) & $P$ & Mean & (s.e.) & $\mathbf{P}$ & Mean & (s.e.) & $\mathbf{P}$ \\
\hline $\begin{array}{c}C R P, m^{\prime-1, a} \\
\text { Total sample } \\
\text { Unadjusted }^{\text {Adjusted }} \\
\text { Adjusted }^{\mathrm{c}}\end{array}$ & $\begin{array}{l}1.09 \\
1.17 \\
1.27\end{array}$ & $\begin{array}{c}N=494 \\
(1.06) \\
(1.06) \\
(1.05)\end{array}$ & $\begin{array}{l}\text { Ref } \\
\text { Ref } \\
\text { Ref }\end{array}$ & $\begin{array}{l}1.25 \\
1.24 \\
1.26\end{array}$ & $\begin{array}{c}N=789 \\
(1.04) \\
(1.04) \\
(1.04)\end{array}$ & $\begin{array}{l}0.05 \\
0.38 \\
0.91\end{array}$ & $\begin{array}{l}1.40 \\
1.36 \\
1.30\end{array}$ & $\begin{array}{r}N=1132 \\
(1.04) \\
(1.04) \\
(1.03)\end{array}$ & $\begin{array}{c}<0.001 \\
0.02 \\
0.66\end{array}$ \\
\hline $\begin{array}{l}\text { Men } \\
\text { Unadjusted } \\
\text { Adjusted }^{\mathrm{b}} \\
\text { Adjusted }^{\mathrm{c}}\end{array}$ & $\begin{array}{l}0.83 \\
0.92 \\
1.04\end{array}$ & $\begin{array}{c}N=193 \\
(1.09) \\
(1.08) \\
(1.08)\end{array}$ & $\begin{array}{l}\text { Ref } \\
\text { Ref } \\
\text { Ref }\end{array}$ & $\begin{array}{l}1.14 \\
1.09 \\
1.03\end{array}$ & $\begin{array}{c}N=238 \\
(1.08) \\
(1.08) \\
(1.07)\end{array}$ & $\begin{array}{l}0.005 \\
0.13 \\
0.96\end{array}$ & $\begin{array}{l}1.36 \\
1.33 \\
1.29\end{array}$ & $\begin{array}{c}N=369 \\
(1.06) \\
(1.06) \\
(1.06)\end{array}$ & $\begin{array}{c}<0.001 \\
<0.001 \\
0.02\end{array}$ \\
\hline $\begin{array}{l}\text { Women } \\
\text { Unadjusted } \\
\text { Adjusted }^{\mathrm{b}} \\
\text { Adjusted }^{\mathrm{C}}\end{array}$ & $\begin{array}{l}1.29 \\
1.36 \\
1.45\end{array}$ & $\begin{array}{c}N=301 \\
(1.07) \\
(1.07) \\
(1.07)\end{array}$ & $\begin{array}{l}\text { Ref } \\
\text { Ref } \\
\text { Ref }\end{array}$ & $\begin{array}{l}1.30 \\
1.34 \\
1.38\end{array}$ & $\begin{array}{c}N=551 \\
(1.05) \\
(1.05) \\
(1.05)\end{array}$ & $\begin{array}{l}0.94 \\
0.82 \\
0.57\end{array}$ & $\begin{array}{l}1.42 \\
1.36 \\
1.30\end{array}$ & $\begin{array}{c}N=763 \\
(1.05) \\
(1.05) \\
(1.04)\end{array}$ & $\begin{array}{l}0.29 \\
0.96 \\
0.16\end{array}$ \\
\hline $\begin{array}{l}\text { IL-6, pg m/ 1, a } \\
\text { Total sample } \\
\text { Unadjusted } \\
\text { Adjusted }^{\mathrm{b}} \\
\text { Adjusted }^{\mathrm{C}}\end{array}$ & $\begin{array}{l}0.72 \\
0.74 \\
0.78\end{array}$ & $\begin{array}{c}N=494 \\
(1.04) \\
(1.04) \\
(1.04)\end{array}$ & $\begin{array}{l}\text { Ref } \\
\text { Ref } \\
\text { Ref }\end{array}$ & $\begin{array}{l}0.75 \\
0.74 \\
0.74\end{array}$ & $\begin{array}{c}N=789 \\
(1.03) \\
(1.03) \\
(1.03)\end{array}$ & $\begin{array}{l}0.49 \\
0.88 \\
0.42\end{array}$ & $\begin{array}{l}0.80 \\
0.79 \\
0.77\end{array}$ & $\begin{array}{c}N=1132 \\
(1.03) \\
(1.03) \\
(1.03)\end{array}$ & $\begin{array}{l}0.04 \\
0.24 \\
0.91\end{array}$ \\
\hline $\begin{array}{l}\text { Men } \\
\text { Unadjusted } \\
\text { Adjusted }^{\mathrm{b}} \\
\text { Adjusted }^{\mathrm{C}}\end{array}$ & $\begin{array}{l}0.68 \\
0.72 \\
0.76\end{array}$ & $\begin{array}{l}N=193 \\
(1.07) \\
(1.07) \\
(1.07)\end{array}$ & $\begin{array}{l}\text { Ref } \\
\text { Ref } \\
\text { Ref }\end{array}$ & $\begin{array}{l}0.82 \\
0.80 \\
0.78\end{array}$ & $\begin{array}{c}N=238 \\
(1.06) \\
(1.06) \\
(1.06)\end{array}$ & $\begin{array}{l}0.03 \\
0.20 \\
0.72\end{array}$ & $\begin{array}{l}0.89 \\
0.88 \\
0.87\end{array}$ & $\begin{array}{c}N=369 \\
(1.05) \\
(1.05) \\
(1.05)\end{array}$ & $\begin{array}{l}0.001 \\
0.01 \\
0.10\end{array}$ \\
\hline $\begin{array}{l}\text { Women } \\
\text { Unadjusted } \\
\text { Adjusted }^{\mathrm{b}} \\
\text { Adjusted }^{\mathrm{C}}\end{array}$ & $\begin{array}{l}0.74 \\
0.77 \\
0.80\end{array}$ & $\begin{array}{c}N=301 \\
(1.06) \\
(1.06) \\
(1.06)\end{array}$ & $\begin{array}{l}\text { Ref } \\
\text { Ref } \\
\text { Ref }\end{array}$ & $\begin{array}{l}0.71 \\
0.71 \\
0.72\end{array}$ & $\begin{array}{c}N=551 \\
(1.04) \\
(1.04) \\
(1.04)\end{array}$ & $\begin{array}{l}0.55 \\
0.22 \\
0.13\end{array}$ & $\begin{array}{l}0.75 \\
0.75 \\
0.73\end{array}$ & $\begin{array}{c}N=763 \\
(1.04) \\
(1.03) \\
(1.03)\end{array}$ & $\begin{array}{l}0.86 \\
0.63 \\
0.14\end{array}$ \\
\hline 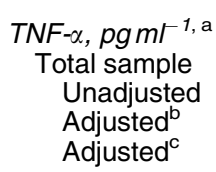 & $\begin{array}{l}0.82 \\
0.84 \\
0.85\end{array}$ & $\begin{array}{c}N=494 \\
(1.03) \\
(1.03) \\
(1.03)\end{array}$ & $\begin{array}{l}\text { Ref } \\
\text { Ref } \\
\text { Ref }\end{array}$ & $\begin{array}{l}0.82 \\
0.82 \\
0.82\end{array}$ & $\begin{array}{c}N=789 \\
(1.02) \\
(1.02) \\
(1.02)\end{array}$ & $\begin{array}{l}0.99 \\
0.60 \\
0.43\end{array}$ & $\begin{array}{l}0.86 \\
0.85 \\
0.84\end{array}$ & $\begin{array}{r}N=1132 \\
(1.02) \\
(1.02) \\
(1.02)\end{array}$ & $\begin{array}{l}0.23 \\
0.59 \\
0.78\end{array}$ \\
\hline $\begin{array}{l}\text { Men } \\
\text { Unadjusted } \\
\text { Adjusted }^{\mathrm{b}} \\
\text { Adjusted }^{\mathrm{c}}\end{array}$ & $\begin{array}{l}0.84 \\
0.85 \\
0.88\end{array}$ & $\begin{array}{l}N=193 \\
(1.04) \\
(1.04) \\
(1.04)\end{array}$ & $\begin{array}{l}\text { Ref } \\
\text { Ref } \\
\text { Ref }\end{array}$ & $\begin{array}{l}0.84 \\
0.83 \\
0.83\end{array}$ & $\begin{array}{c}N=238 \\
(1.04) \\
(1.04) \\
(1.04)\end{array}$ & $\begin{array}{l}0.90 \\
0.65 \\
0.33\end{array}$ & $\begin{array}{l}0.87 \\
0.87 \\
0.86\end{array}$ & $\begin{array}{c}N=369 \\
(1.03) \\
(1.03) \\
(1.03)\end{array}$ & $\begin{array}{l}0.41 \\
0.70 \\
0.69\end{array}$ \\
\hline $\begin{array}{l}\text { Women } \\
\text { Unadjusted } \\
\text { Adjusted }^{\mathrm{b}} \\
\text { Adjusted }^{\mathrm{c}}\end{array}$ & $\begin{array}{l}0.81 \\
0.83 \\
0.84\end{array}$ & $\begin{array}{c}N=301 \\
(1.04) \\
(1.04) \\
(1.04)\end{array}$ & $\begin{array}{l}\text { Ref } \\
\text { Ref } \\
\text { Ref }\end{array}$ & $\begin{array}{l}0.81 \\
0.81 \\
0.82\end{array}$ & $\begin{array}{c}N=551 \\
(1.03) \\
(1.03) \\
(1.03)\end{array}$ & $\begin{array}{l}0.97 \\
0.76 \\
0.70\end{array}$ & $\begin{array}{l}0.85 \\
0.84 \\
0.83\end{array}$ & $\begin{array}{c}N=763 \\
(1.02) \\
(1.02) \\
(1.02)\end{array}$ & $\begin{array}{l}0.34 \\
0.74 \\
0.90\end{array}$ \\
\hline
\end{tabular}

Abbreviations: CRP, C-reactive protein; IL-6, interleukin-6; TNF- $\alpha$, tumor necrosis factor-alpha.

${ }^{a}$ To normalize distributions CRP, IL- 6 and TNF- $\alpha$ were In-transformed; for interpretation purposes presented means were back transformed.

bBased on analyses of (co)variance; adjusted for sex, age and education.

${ }^{\mathrm{c} A d d i t i o n a l l y ~ a d j u s t e d ~ f o r ~ s m o k i n g ~ s t a t u s, ~ a l c o h o l ~ i n t a k e, ~ b o d y ~ m a s s ~ i n d e x, ~ p h y s i c a l ~ a c t i v i t y, ~ c a r d i o v a s c u l a r ~ d i s e a s e, ~ d i a b e t e s, ~ n u m b e r ~ o f ~ o t h e r ~ c h r o n i c ~ d i s e a s e s, ~}$ statins and anti-inflammatory medication.

Table 3 Association $^{\mathrm{a}}$ of depression characteristics with inflammatory markers in persons with current depressive disorders $(N=1132)$

\begin{tabular}{|c|c|c|c|c|c|c|}
\hline \multirow[b]{2}{*}{ Depression characteristic } & \multicolumn{2}{|c|}{$C R P^{b}$} & \multicolumn{2}{|c|}{$I L-6^{b}$} & \multicolumn{2}{|c|}{$T N F-\alpha^{\mathrm{b}}$} \\
\hline & $\beta$ & $P$ & $\beta$ & $\mathrm{P}$ & $\beta$ & $P$ \\
\hline $\begin{array}{l}\text { Severity (IDS score) } \\
\text { Men } \\
\text { Women }\end{array}$ & -0.020 & 0.47 & 0.038 & 0.22 & $\begin{array}{r}0.040 \\
-0.048 \\
0.085\end{array}$ & $\begin{array}{l}0.20 \\
0.36 \\
0.02\end{array}$ \\
\hline Duration of depressive symptoms & -0.005 & 0.87 & -0.017 & 0.58 & 0.012 & 0.70 \\
\hline $\begin{array}{l}\text { Age of depression onset } \\
\text { Men } \\
\text { Women }\end{array}$ & $\begin{array}{l}0.064 \\
0.140 \\
0.016\end{array}$ & $\begin{array}{l}0.04 \\
0.003 \\
0.67\end{array}$ & -0.002 & 0.95 & $\begin{array}{r}0.043 \\
0.149 \\
-0.025\end{array}$ & $\begin{array}{l}0.22 \\
0.004 \\
0.55\end{array}$ \\
\hline
\end{tabular}

Abbreviations: CRP, C-reactive protein; IDS, inventory of depressive symptoms; IL-6, interleukin-6; TNF- $\alpha$, tumor necrosis factor-alpha.

aBased on linear regression analyses adjusted for (sex), age, education, smoking status, alcohol intake, body mass index, physical activity, cardiovascular disease, diabetes, number of other chronic diseases, statins, anti-inflammatory medication; sex-specific associations are shown in case $P$ sex interaction $\leqslant 0.05$

${ }^{\mathrm{b}} \mathrm{CRP}, \mathrm{IL}-6$ and TNF- $\alpha$ were In-transformed to normalize distributions. 
control group of medication-free depressed persons with a current diagnosis and an IDS score $\geqslant 25(N=426)$. In this medication-free reference group the mean IDS score was comparable to the other medication groups. As effects of TCA and TeCA users were comparable, they were grouped together to increase numbers. Sex interactions in the association between antidepressant medication group (no medication, SSRI, SNRI and TCA/TeCA) and inflammatory markers were found (CRP: $P$-interaction $=0.06$, IL-6: $P$-interaction $=0.02 ;$ TNF- $\alpha$ : $P$-interaction $=0.90)$. Therefore, Figure 1 shows adjusted mean inflammation levels comparing antidepressant users with medication-free persons, for men and women separately. Increased levels of CRP were found for men using SNRI (1.98 versus $1.21 \mathrm{mgl}^{-1}, P=0.02$, Cohen's $\mathrm{d}=0.44$ ) or TCA/TeCA (2.05 versus $1.21 \mathrm{mgl}^{-1}$, $P=0.02$, Cohen's $\mathrm{d}=0.48$ ) as compared with medicationfree depressed men. A trend for higher CRP in TCA/TeCA users was also found for women (2.10 versus $1.53 \mathrm{mgl}^{-1}$, $P=0.08$, Cohen's $\mathrm{d}=0.28)$. In men only, IL-6 levels were lower in SSRI users (0.70 versus $0.91 \mathrm{pg} \mathrm{ml}^{-1}, P=0.02$, Cohen's $d=-0.32$ ) and considerably increased in SNRI users (1.37 versus $0.91 \mathrm{pg} \mathrm{ml}^{-1}, P=0.01$, Cohen's $\mathrm{d}=0.49$ ) compared with medication-free depressed men. No associations were found for TNF- $\alpha$. For comparison, inflammation levels in persons with cardiovascular disease were $1.84 \mathrm{mgl}^{-1}$ for CRP and $1.06 \mathrm{pg} \mathrm{ml}^{-1}$ for IL-6, suggesting that the putative effects of antidepressant medication are at least of similar magnitude as having cardiovascular disease. As antidepressant medication effects were found, we checked whether these influenced the findings from Tables 2 and 3, but results remained similar after additional adjustment for antidepressant medication group.

\section{Discussion}

The present study examined the association between depressive disorders, depression characteristics and antidepressant medication with inflammation in a large cohort of depressed persons and controls. After taking a large set of possibly confounding factors into account, it was found that men with current depressive disorders had higher levels of CRP, and marginally higher levels of IL-6, but not of TNF- $\alpha$. No overall associations were found in women. Increased inflammation was in particular found in depressed men with an older age of depression onset (CRP, TNF- $\alpha$ ). Users of SNRI (men only), TCA and TeCA had increased levels of CRP and IL-6, whereas men using SSRI had significantly lower levels of IL-6.

Our results confirm previous findings of immune dysregulation in depressed persons. ${ }^{3,4}$ In men, effect sizes for current depression were small to moderate for both CRP and IL-6. These effect sizes are comparable to those reported by the meta-analysis of Howren, ${ }^{4}$ in which stronger effects were found for studies using clinical interviews compared with studies using self-report questionnaires. Compared with symptom questionnaires, a depressive disorder diagnosis is less confounded by somatic health problems. Together with the fact that our results were elaborately adjusted for possibly confounding factors, our findings suggest a true relationship between depression and inflammation in men.
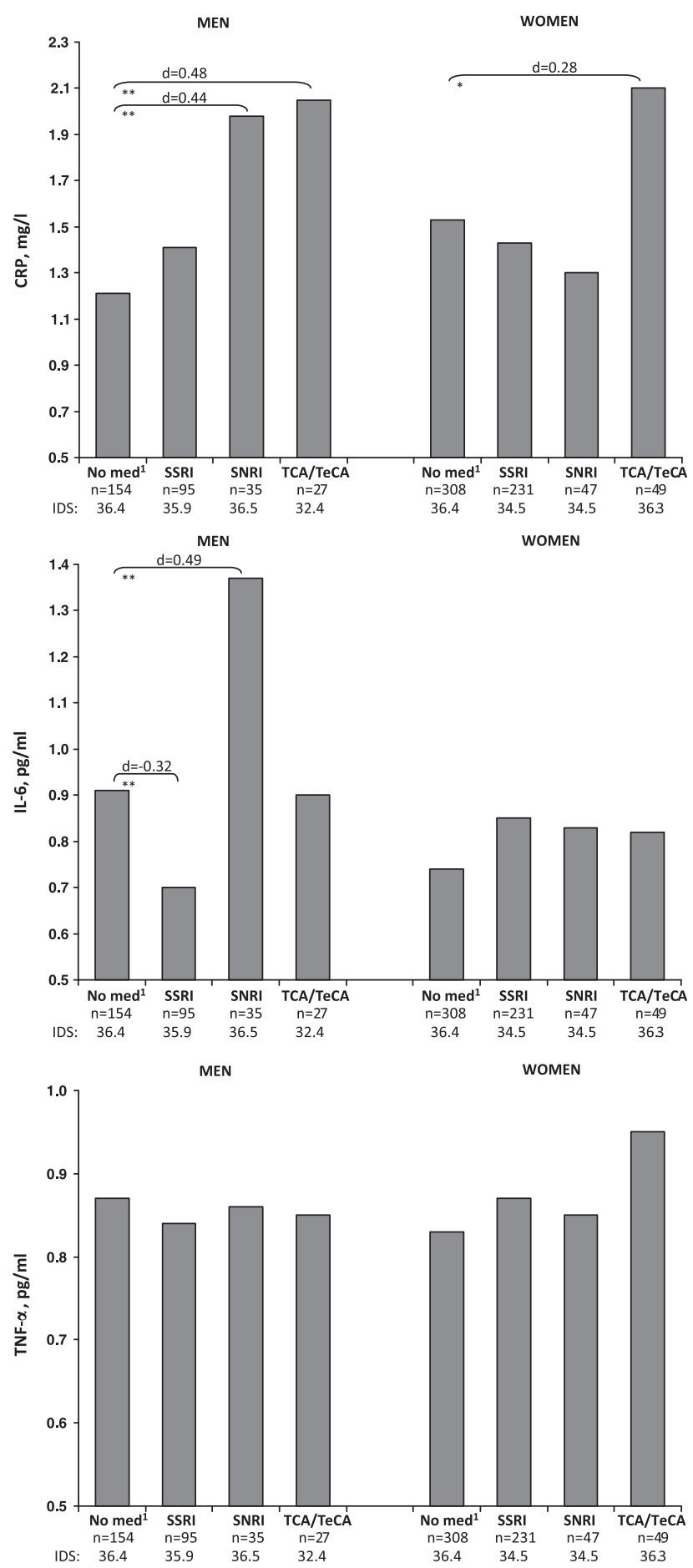

Figure 1 Adjusted mean inflammatory levels across medication groups and sex based on analyses of covariance (ANCOVA) adjusted for age, education, smoking status, alcohol intake, body mass index (BMI), physical activity, cardiovascular disease, diabetes, number of other chronic diseases, statins and anti-inflammatory medication; to normalize distributions C-reactive protein (CRP), Interleukin-6 (IL-6) and Tumor necrosis factor-alpha (TNF- $\alpha$ ) were In-transformed, for interpretation purposes presented means were back transformed. Abbreviations: IDS, inventory of depressive symptoms score; SNRI, serotonin-norepinephrine reuptake inhibitor; SSRI, selective serotonin reuptake inhibitor; TCA, tricyclic antidepressant; TeCA, tetracyclic antidepressant. ${ }^{1}$ Current depressive disorder and IDS $\geqslant 25$ without medication use (= reference); only significant differences from reference are shown: ${ }^{*} P<0.10 ;{ }^{*} P<0.05$. 
No clear associations between depression and inflammation were found in women. This is in line with results from another large and relatively young cohort study in which history of depression was associated with CRP levels in men, but not women. ${ }^{5}$ Hormonal changes throughout female life due to phase of menstrual cycle, use of hormonal contraceptives, menopause and use of estrogens greatly impact on inflammation levels, ${ }^{6-8}$ which could confound a clear association between depression and inflammation. However, in our study, associations between depression and inflammation appeared absent in both pre- and postmenopausal women and adjusting for several hormonal factors did not change our findings. Another explanation might be that in women, psychosocial factors have a larger role in depression and therefore override the effects of biological factors. For instance, insufficient social support and stressful life events have been found to pose a greater risk for depression among women compared with men. ${ }^{26,27}$

Results of increased inflammation were particularly present in men with late-onset depression. In contrast, characteristics that are more often associated with an early age of onset, such as higher severity and longer duration, were not consistently associated with increased inflammation. Interesting to note is that women in our study also had an earlier age of depression onset compared with men. A distinct etiology in late-onset versus early-onset depression was also found by Kendler et al. ${ }^{28}$ This study showed that depression with an early age of onset was associated with a family history of depression, whereas late-onset depression was associated with a family history of vascular disease. Also, subclinical vascular dysregulations, such as atherosclerosis, have been found to relate to late-onset depression. ${ }^{11,12}$ These findings are in line with the vascular depression hypothesis, which suggests that vascular damage in the brain predisposes to late-onset depression. ${ }^{29}$ As immune dysregulation is critically involved in vascular disease ${ }^{13}$ this vascular damage could be the result of increased inflammation.

Instead of inflammatory or vascular, the true etiology of depression in this subgroup of men with late-onset depression might have a metabolic nature. Several studies have confirmed an association between CRP, IL- 6 and TNF- $\alpha$ with the metabolic syndrome and visceral fat depots release cytokines. $^{30}$ The metabolic syndrome and in particular visceral fat have been bidirectionally associated with depression in late life, ${ }^{31,32}$ specifically in men. Men possess higher amounts of visceral fat compared with women and are therefore more likely to experience related inflammation and depression. Involvement of metabolic processes is further supported by our finding that the association between depression and inflammation in particular decreased after adjustment for BMI.

Several biological mechanisms could further explain the relationship between depression and inflammation. Depression has been associated with dysregulation of important stress systems of the human body, that is, the hypothalamuspituitary-adrenal-axis ${ }^{33}$ and the autonomic nervous system. ${ }^{34}$ Although the hypothalamus-pituitary-adrenal-axis in normal situations should temper inflammatory reactions, prolonged hyperactivity of the hypothalamus-pituitary-adrenal-axis could result in blunted anti-inflammatory responses to glucocorticoids resulting in increased inflammation. ${ }^{35,36}$ In addition, both decreased parasympathetic ${ }^{37}$ as well as increased sympathetic nervous system activity ${ }^{38}$ have been associated with increased inflammation. Furthermore, proinflammatory cytokines might inhibit hippocampal neurogenesis, ${ }^{39}$ which could lead to a reduced hippocampal volume, ${ }^{40}$ which is also seen in depression. ${ }^{41}$ Also, several inflammatory markers have been shown to promote indoleamine-2,3dioxygenase activation, ${ }^{42}$ which catalyzes tryptophan, the precursor of serotonin, to kynurenine, thereby indirectly reducing the availability of serotonin. ${ }^{43}$ Lastly, specific genes might underlie both increased inflammation and depression, as several inflammation-related genes have been associated with susceptibility to major depression. ${ }^{44}$

Our study also shows that inflammation levels differ across persons using different types of antidepressant medication. The highest inflammation levels were found in men using SNRI, TCA or TeCA, while IL-6 levels in men using SSRI were lower compared with medication-free depressed men. Interesting to note is that the first three classes of medication have a combined serotonergic/noradrenergic effect, whereas SSRI act purely serotonergic. Earlier studies found decreases in inflammatory marker levels after antidepressant treatment, mainly SSRI (see ref. 14 for an overview), whereas two recent large studies found that use of antidepressants, mainly TCA, was associated with elevated inflammation levels. ${ }^{15}$ Possibly, noradrenergic effects are driving increased inflammation mechanisms. Noradrenaline is a part of the human stress response and has been suggested to potentiate cytokine production. ${ }^{45}$ Use of SNRI, TCA and TeCA has also been observed to disturb functioning of autonomic nervous system, ${ }^{46}$ blood pressure ${ }^{47}$ and the metabolic syndrome. ${ }^{48}$ Although it is possible that persons using SNRI, TCA or TeCA are in other ways different from SSRI users and medication-free depressed persons, we constructed our groups in such a way that depression severity levels were very comparable. In addition, we adjusted our analyses for a large set of covariates and have therefore taken possible differences in lifestyle or disease factors into account. On the other hand, evidence suggests that increased inflammatory activity before treatment predicts non-response. ${ }^{49,50}$ Possibly, persons with elevated inflammation did not respond to SSRI and were therefore prescribed SNRI, TCA or TeCA.

Associations found in this study were not always consistent across all inflammatory markers. Correlations between inflammatory markers were only modest and were highest between CRP and IL-6. Regulation of the immune system is rather complex and involves many different inflammatory mechanisms. Most consistent findings were found for CRP, which is a very general marker of inflammation. IL- 6 and TNF- $\alpha$, on the other hand, only tap part of the immune system. This seems to suggest that inflammation is indeed involved in depression, but it is still unclear which part of the immune system is most critically involved. Nonetheless, expected associations with covariates (for example, age, smoking, alcohol use, BMI, physical activity and somatic disease (medication)) were found for all inflammatory markers.

What do the findings of our study implicate with regard to treatment of depression? Considering the heterogeneity of depression and the fact that current treatment of depression is 
only effective in about a third to a half of patients, ${ }^{1,2}$ this indicates that new treatments are needed for specific subgroups. Our finding of increased inflammation in men with late-onset depression, together with previous findings of high inflammation indicating non-response to antidepressants, ${ }^{49,50}$ might suggest that this specific subgroup could benefit from alternative treatments, with anti-inflammatory medication being a likely candidate. Preliminary evidence from studies among patients treated with anti-inflammatory agents for other indications suggests that these agents may have beneficial effects on mood. ${ }^{51}$ One study found positive effects on mood in medically healthy, major depressed patients. ${ }^{52}$ On the other hand we found, like others, ${ }^{14}$ that SSRI might have a beneficial effect on inflammation, suggesting that SSRI could be effective in depressed patients with immune dysregulation through this anti-inflammatory effect. Furthermore, behavioral interventions, such as exercise, have been shown to normalize immune and metabolic dysregulation, ${ }^{53}$ as well as to improve depressive symptoms to some degree, ${ }^{54}$ and might therefore be an indicated treatment for a immune/metabolic depression subgroup. At this moment, these considerations for treatment implications are still very speculative. Follow-up (longitudinal) research should confirm and further delineate an inflammatory (or metabolic) depression subtype, taking into account age, sex, depression characteristics and course. Experimental studies are needed to examine and compare the effects of different types of currently available antidepressants, antiinflammatory medication and behavioral (exercise) interventions on both immune/metabolic parameters and depression.

Our study has some important strengths such as a large sample size, assessment of multiple inflammatory markers, clinical diagnoses of depression, adequate adjustment for potential confounders, the ability to examine the role of depression characteristics and antidepressant medication. However, some limitations need to be acknowledged. As our data are cross-sectional, we cannot make any inferences about the direction of the association. Longitudinal studies are needed to investigate whether immune dysregulation is a precursor or the result of depression (treatment), or whether this relationship is bidirectional. The few available prospective studies have shown contradicting results. ${ }^{9,55,56}$ Further, like most other studies, we assessed circulating levels of inflammatory markers, which show a high degree of intra-individual variation, which could explain the rather modest overall associations between depression and inflammation in our study.

In conclusion, our study suggests that immune dysregulation has a role in a subgroup of depressed persons, in particular in men with a late-onset depression. Treatment trials should further examine the differential effects of different types of antidepressants on inflammation. Whether a specific treatment strategy (SSRI, anti-inflammatory drugs, exercise) is indicated for a subgroup of late-onset depressed patients with immune dysregulation needs to be further investigated, using longitudinal and experimental study designs.

\section{Conflict of interest}

The authors declare no conflict of interest.
Acknowledgements. The infrastructure for the NESDA study (www.nesda.nl) is funded through the Geestkracht program of the Netherlands Organization for Health Research and Development (Zon-Mw, grant number 10000-1002) and is supported by participating universities and mental health care organizations (VU University Medical Center, GGZ inGeest, Arkin, Leiden University Medical Center, GGZ Rivierduinen, University Medical Center Groningen, Lentis, GGZ Friesland, GGZ Drenthe, Institute for Quality of Health Care (IQ Healthcare), Netherlands Institute for Health Services Research (NIVEL) and Netherlands Institute of Mental Health and Addiction (Trimbos). Data analyses were supported through a fellowship (NV) from the EMGO Institute for Health and Care Research. Assaying of inflammatory markers was supported by the Neuroscience Campus Amsterdam.

1. Pigott HE, Leventhal AM, Alter GS, Boren JJ. Efficacy and effectiveness of antidepressants: current status of research. Psychother Psychosom 2010; 79: 267-279.

2. Rush AJ, Trivedi MH, Wisniewski SR, Nierenberg AA, Stewart JW, Warden D et al. Acute and longer-term outcomes in depressed outpatients requiring one or several treatment steps: a STAR ${ }^{\star}$ D report. Am J Psychiatry 2006; 163: 1905-1917.

3. Dowlati Y, Herrmann N, Swardfager W, Liu H, Sham L, Reim EK et al. A meta-analysis of cytokines in major depression. Biol Psychiatry 2010; 67: 446-457.

4. Howren MB, Lamkin DM, Suls J. Associations of depression with C-reactive protein, IL-1, and IL-6: a meta-analysis. Psychosom Med 2009; 71: 171-186.

5. Danner M, Kasl SV, Abramson JL, Vaccarino V. Association between depression and elevated C-reactive protein. Psychosom Med 2003; 65: 347-356.

6. Cushman M, Legault C, Barrett-Connor E, Stefanick ML, Kessler C, Judd HL et al. Effect of postmenopausal hormones on inflammation-sensitive proteins: the Postmenopausal Estrogen/Progestin Interventions (PEPI) Study. Circulation 1999; 100: 717-722.

7. Dreon DM, Slavin JL, Phinney SD. Oral contraceptive use and increased plasma concentration of C-reactive protein. Life Sci 2003; 73: 1245-1252.

8. Jilma B, Dirnberger E, Loscher I, Rumplmayr A, Hildebrandt J, Eichler HG et al. Menstrual cycle-associated changes in blood levels of interleukin-6, alpha1 acid glycoprotein, and Creactive protein. J Lab Clin Med 1997; 130: 69-75.

9. Duivis HE, de JP, Penninx BW, Na BY, Cohen BE, Whooley MA. Depressive symptoms, health behaviors, and subsequent inflammation in patients with coronary heart disease: prospective findings from the heart and soul study. Am J Psychiatry 2011; 168: 913-920.

10. Hamer M, Molloy GJ, de OC, Demakakos P. Persistent depressive symptomatology and inflammation: to what extent do health behaviours and weight control mediate this relationship? Brain Behav Immun 2009; 23: 413-418.

11. Seldenrijk A, van Hout HP, van Marwijk HW, de GE, Gort J, Rustemeijer $C$ et al. Carotid atherosclerosis in depression and anxiety: Associations for age of depression onset. World J Biol Psychiatry 2011; 12: 549-558.

12. Smith PJ, Blumenthal JA, Babyak MA, Doraiswamy PM, Hinderliter A, Hoffman BM et al. Intima-media thickness and age of first depressive episode. Biol Psychol 2009; 80: 361-364.

13. Willerson JT, Ridker PM. Inflammation as a cardiovascular risk factor. Circulation 2004; 109(21 Suppl 1): ॥2-II10

14. Miller AH, Maletic V, Raison CL. Inflammation and its discontents: the role of cytokines in the pathophysiology of major depression. Biol Psychiatry 2009; 65: 732-741.

15. Hamer M, Batty GD, Marmot MG, Singh-Manoux A, Kivimaki M. Anti-depressant medication use and C-reactive protein: results from two population-based studies. Brain Behav Immun 2011; 25: 168-173.

16. Penninx BW, Beekman AT, Smit JH, Zitman FG, Nolen WA, Spinhoven $P$ et al. The Netherlands Study of Depression and Anxiety (NESDA): rationale, objectives and methods. Int J Methods Psychiatr Res 2008; 17: 121-140.

17. American Psychiatric Association. Diagnostic and statistical manual of mental disorders, fourth edition. 4th edn. American Psychiatric Association: Washington, DC, 2001.

18. Wittchen HU. Reliability and validity studies of the WHO-Composite International Diagnostic Interview (CIDI): a critical review. J Psychiatr Res 1994; 28: 57-84.

19. Rush AJ, Gullion CM, Basco MR, Jarrett RB, Trivedi MH. The Inventory of Depressive Symptomatology (IDS): psychometric properties. Psychol Med 1996; 26: 477-486.

20. Lyketsos CG, Nestadt G, Cwi J, Heithoff K, Eaton WW. The life chart interview: A standardized method to describe the course of psychopathology. Int $J$ Methods Psychiatr Res 1994; 4: 143-155

21. WHO Collaborating Centre for Drug Statistics Methodology. Anatomical Therapeutic Chemical Classification. World Health Organization: Geneva, 2007.

22. Stuurgroep Multidisciplinaire Richtlijnontwikkeling. Stoornissen in het gebruik van alcohol: Richtlijn voor de diagnostiek en behandeling van patiënten met een stoornis in het gebruik van alcohol. GGZ: Utrecht, The Netherlands, 2009.

23. Gianoulakis C, Dai X, Brown T. Effect of chronic alcohol consumption on the activity of the hypothalamic-pituitary-adrenal axis and pituitary beta-endorphin as a function of alcohol intake, age, and gender. Alcohol Clin Exp Res 2003; 27: 410-423.

24. Craig CL, Marshall AL, Sjostrom M, Bauman AE, Booth ML, Ainsworth BE et al. International physical activity questionnaire: 12-country reliability and validity. Med Sci Sports Exerc 2003; 35: 1381-1395. 
25. Vogelzangs N, Seldenrijk A Beekman AT, van Hout HP, de JP, Penninx BW. Cardiovascular disease in persons with depressive and anxiety disorders. J Affect Disord 2010; 125: 241-248

26. Kendler KS, Myers J, Prescott CA. Sex differences in the relationship between social support and risk for major depression: a longitudinal study of opposite-sex twin pairs. Am J Psychiatry 2005; 162: 250-256.

27. Maciejewski PK, Prigerson HG, Mazure CM. Sex differences in event-related risk for major depression. Psychol Med 2001; 31: 593-604

28. Kendler KS, Fiske A, Gardner CO, Gatz M. Delineation of two genetic pathways to major depression. Biol Psychiatry 2009; 65: 808-811.

29. Alexopoulos GS, Meyers BS, Young RC, Campbell S, Silbersweig D, Charlson M. Vascular depression' hypothesis. Arch Gen Psychiatry 1997; 54: 915-922.

30. Sutherland JP, McKinley B, Eckel RH. The metabolic syndrome and inflammation. Metab Syndr Relat Disord 2004; 2: 82-104.

31. Vogelzangs N, Kritchevsky SB, Beekman AT, Newman AB, Satterfield S, Simonsick EM et al. Depressive symptoms and change in abdominal obesity in older persons. Arch Gen Psychiatry 2008; 65: 1386-1393.

32. Vogelzangs N, Kritchevsky SB, Beekman AT, Brenes GA, Newman AB, Satterfield S et al. Obesity and onset of significant depressive symptoms: results from a prospective communitybased cohort study of older men and women. J Clin Psychiatry 2010; 71: 391-399.

33. Vreeburg SA, Hoogendijk WJ, van Pelt J, Derijk RH, Verhagen JC, Van DR et al. Major depressive disorder and hypothalamic-pituitary-adrenal axis activity: results from a large cohort study. Arch Gen Psychiatry 2009; 66: 617-626.

34. Licht CM, de Geus EJ, Zitman FG, Hoogendijk WJ, Van DR, Penninx BW. Association between major depressive disorder and heart rate variability in the Netherlands Study of Depression and Anxiety (NESDA). Arch Gen Psychiatry 2008; 65: 1358-1367.

35. Miller GE, Cohen S, Ritchey AK. Chronic psychological stress and the regulation of proinflammatory cytokines: a glucocorticoid-resistance model. Health Psychol 2002: 21: 531-541.

36. Wirtz PH, von KR, Schnorpfeil P, Ehlert U, Frey K, Fischer JE. Reduced glucocorticoid sensitivity of monocyte interleukin- 6 production in male industrial employees who are vitally exhausted. Psychosom Med 2003; 65: 672-678.

37. Sloan RP, McCreath H, Tracey KJ, Sidney S, Liu K, Seeman T. RR interval variability is inversely related to inflammatory markers: the CARDIA study. Mol Med 2007; 13: 178-184.

38. Johnson JD, Campisi J, Sharkey CM, Kennedy SL, Nickerson M, Greenwood BN et al. Catecholamines mediate stress-induced increases in peripheral and central inflammatory cytokines. Neuroscience 2005; 135: 1295-1307.

39. Ekdahl CT, Claasen $\mathrm{JH}$, Bonde $\mathrm{S}$, Kokaia Z, Lindvall $\mathrm{O}$. Inflammation is detrimental for neurogenesis in adult brain. Proc Natl Acad Sci USA 2003; 100: 13632-13637.

40. Marsland AL, Gianaros PJ, Abramowitch SM, Manuck SB, Hariri AR. Interleukin- 6 covaries inversely with hippocampal grey matter volume in middle-aged adults. Biol Psychiatry 2008; 64: 484-490.

41. Campbell S, Marriott M, Nahmias C, MacQueen GM. Lower hippocampal volume in patients suffering from depression: a meta-analysis. Am J Psychiatry 2004; 161: 598-607.

42. Fujigaki H, Saito K, Fujigaki S, Takemura M, Sudo K, Ishiguro H et al. The signal transduce and activator of transcription 1 alpha and interferon regulatory factor 1 are not essential for the induction of indoleamine 2,3-dioxygenase by lipopolysaccharide: involvement of p38 mitogen-activated protein kinase and nuclear factor-kappaB pathways, and synergistic effect of several proinflammatory cytokines. J Biochem 2006; 139: 655-662.
43. Dantzer R, O'Connor JC, Freund GG, Johnson RW, Kelley KW. From inflammation to sickness and depression: when the immune system subjugates the brain. Nat Rev Neurosci 2008; 9: 46-56.

44. Wong ML, Dong C, Maestre-Mesa J, Licinio J. Polymorphisms in inflammation-related genes are associated with susceptibility to major depression and antidepressant response. Mol Psychiatry 2008; 13: 800-812.

45. Elenkov IJ, Chrousos GP. Stress hormones, proinflammatory and antiinflammatory cytokines, and autoimmunity. Ann N Y Acad Sci 2002; 966: 290-303.

46. Licht CM, de Geus EJ, Van DR, Penninx BW. Longitudinal evidence for unfavorable effects of antidepressants on heart rate variability. Biol Psychiatry 2010; 68: 861-868.

47. Licht CM, de Geus EJ, Seldenrijk A, van Hout HP, Zitman FG, Van DR et al. Depression is associated with decreased blood pressure, but antidepressant use increases the risk for hypertension. Hypertension 2009; 53: 631-638.

48. van Reedt Dortland AK, Giltay EJ, van VT, Zitman FG, Penninx BW. Metabolic syndrome abnormalities are associated with severity of anxiety and depression and with tricyclic antidepressant use. Acta Psychiatr Scand 2010; 122: 30-39.

49. Lanquillon S, Krieg JC, ing-Abu-Shach $\mathrm{U}$, Vedder $\mathrm{H}$. Cytokine production and treatment response in major depressive disorder. Neuropsychopharmacology 2000; 22: 370-379.

50. Eller T, Vasar V, Shlik J, Maron E. Pro-inflammatory cytokines and treatment response to escitalopram in major depressive disorder. Prog Neuropsychopharmacol Biol Psychiatry 2008; 32: 445-450.

51. Muller N, Riedel M, Schwarz MJ. Psychotropic effects of COX-2 inhibitors-a possible new approach for the treatment of psychiatric disorders. Pharmacopsychiatry 2004; 37: $266-269$.

52. Muller N, Schwarz MJ, Dehning S, Douhe A, Cerovecki A, Goldstein-Muller B et al. The cyclooxygenase-2 inhibitor celecoxib has therapeutic effects in major depression: results of a double-blind, randomized, placebo controlled, add-on pilot study to reboxetine. $\mathrm{Mol}$ Psychiatry 2006; 11: 680-684.

53. You T, Nicklas BJ. Effects of exercise on adipokines and the metabolic syndrome. Curr Diab Rep 2008; 8: 7-11.

54. Mead GE, Morley W, Campbell P, Greig CA, McMurdo M, Lawlor DA. Exercise for depression. Cochrane Database Syst Rev 2009; CD004366.

55. Gimeno D, Kivimaki M, Brunner EJ, Elovainio M, De VR, Steptoe A et al. Associations of Creactive protein and interleukin-6 with cognitive symptoms of depression: 12-year follow-up of the Whitehall II study. Psychol Med 2009; 39: 413-423.

56. Stewart JC, Rand KL, Muldoon MF, Kamarck TW. A prospective evaluation of the directionality of the depression-inflammation relationship. Brain Behav Immun 2009; 23: 936-944.

Translational Psychiatry is an open-access journal published by Nature Publishing Group. This work is licensed under the Creative Commons Attribution-Noncommercial-No Derivative Works 3.0 Unported License. To view a copy of this license, visit http://creativecommons.org/licenses/by-nc-nd/3.0/ 